\title{
Características citológicas previas al diagnóstico de cáncer de cérvix en mujeres de Medellín (Colombia)
}

\author{
Cytological characteristics prior to the diagnosis of cervical cancer in women from Medellin (Colombia) \\ Jhon H. Osorio-Castaño'* orcid.org/0000-0001-6272-722X \\ Marjorie Pérez-Villa' orcid.org/0000-0003-1021-7054 \\ Claudia P. Montoya-Zapata' ${ }^{1}$ orcid.org/0000-0002-5155-6688
}

Fernando A. Cardona-Restrepo' ${ }^{1}$ orcid.org/0000-0002-7059-636X

1. Escuela Ciencias de la Salud, Facultad de Enfermería. Universidad Pontificia Bolivariana. Medellín, Colombia

Osorio-Castaño JH, Pérez-Villa M, Montoya-Zapata CP, Cardona-Restrepo FA. Características citológicas previas al diagnóstico de cáncer de cérvix en mujeres de una institución de Medellín. Univ. Salud. 2020;22(3):231-237. DOI: https://doi.org/10.22267/rus.202203.195

\section{Resumen}

Introducción: Actualmente, el cáncer de cérvix sigue siendo una enfermedad importante por su incidencia y mortalidad. Aunque se conoce su etiología generada por la infección del virus del Papiloma Humano (PVH) y sus métodos de tamizaje como la citología convencional y el test de ADN-PVH, aún es necesario reconocer diversas características que se relacionan con la aparición de este tipo de cáncer. Objetivo: Describir las características citológicas previas al diagnóstico de cáncer cervical en mujeres de una institución de salud de la ciudad de Medellín. Materiales y métodos: Estudio descriptivo. Se tomaron los registros clínicos correspondientes a mujeres con diagnóstico confirmado de cáncer de cérvix entre 2012 y 2017 y se revisaron los resultados de citología previos al diagnóstico. Se realizó análisis univariado para describir las variables de estudio. Resultados: La muestra de mujeres con cáncer fue de 714, la mediana de edad fue 51 años (rango intercuartílico: 42-60), la alteración citológica más frecuente fue lesión intraepitelial de alto grado (33,8\%), el principal cáncer diagnosticado fue carcinoma escamocelular (70,6\%). Conclusiones: Los resultados de citología alteradas son un indicador para riesgo de cáncer de cérvix, de ahí la importancia de continuar con el protocolo de tamizaje establecido.

Palabras clave: Cuello del útero; neoplasias del cuello uterino; colposcopía; citología; lesiones intraepiteliales escamosas de cuello uterino (Fuente: DeCS, Bireme).

\begin{abstract}
Introduction: Currently, cervical cancer continues to be an important disease because of its high incidence and mortality. Even though its etiology (Human Papilloma Virus infection) and screening methods (conventional cytology and HPV-DNA test) are well known, understanding the various characteristics associated with the appearance of cervical cancer is fundamental. Objective: To describe the cytological characteristics that precede the diagnosis of cervical cancer in women diagnosed in a health care institution from the city of Medellín. Materials and methods: A descriptive study. The clinical records corresponding to women with a confirmed diagnosis of cervical cancer between 2012 and 2017 were used. Also, the citology results obtained before the diagnosis were reviewed. An univariate analysis was carried out in oder to describe the study variables. Results: The sample size of women with cervical cancer was 714, the age median was 51 years old (interquartile range: 42-60), the most frequent cytological alteration was high-grade intraepithelial lesion (33.8\%), and the main diagnosed cancer type was squamous cell carcinoma (70.6\%). Conclusions: Results showing an altered cytology are indicators of cervical cancer risk. Therefore, it is important to continue with the stablished screening protocol.
\end{abstract}

Keywords: Cervix uteri; uterine cervical neoplasms; colposcopy; cytology; squamous intraepithelial lesions of the cervix (Source: DeCS, Bireme).

\footnotetext{
*Autor de correspondencia

Marjorie Pérez-Villa

e-mail: marjorie.perez@upb.edu.co
} 


\section{Introducción}

En la actualidad el cáncer aumenta su incidencia con el desarrollo económico e industrial de los últimos años, aunque los sistemas de salud se han perfeccionado para la detección y tratamiento, las neoplasias aparecen con más frecuencia(1). Esto se deriva de múltiples factores de carácter biológico, psicológico y sociocultural, los cuales se comportan de manera específica en cada persona, manifestándose en un tipo particular de cáncer ${ }^{(1,2)}$.

En el cáncer cervicouterino las células empiezan a desarrollarse de manera anormal en presencia de una infección persistente o prolongada por el virus del papiloma humano (PVH), el $90 \%$ se presenta en las células escamosas y se inician en la zona de transformación del exocérvix; el otro $10 \%$ son adenocarcinomas que se presentan en la capa cilíndrica glandular del endocérvix ${ }^{(3)}$.

A nivel mundial se producen alrededor de 530.000 nuevos casos de cáncer cervicouterino cada año y cerca del $80 \%$ corresponde a países en vías de desarrollo. Países Suraméricanos registran las incidencias más altas, seguido de centro América, el Caribe y parte de Asia. Por otra parte, las incidencias más bajas se reportan en Europa, Norteamérica y Japón $(4,5)$.

Según el informe del Global Cancer Observatory (2018), el cáncer de cérvix ocupa el cuarto lugar tanto en incidencia $(3,2 \%)$ como en mortalidad $(3,3 \%)$, con un estimado de 570.000 casos y 311.000 muertes en todo el mundo(6,7); mientras que la Organización Mundial de la Salud informa que para 2018 fueron más de 72.000 mujeres diagnosticadas y que de estas fallecieron 34.000 en el continente americano(3,8). Este tipo de cáncer es uno los principales causantes de la muerte de mujeres entre los 30 y 59 años en Colombia, reportándose para el 2018 una incidencia del 3,8\% y una mortalidad del 3,9\%, además la prevalencia a cinco años para todas las edades del $41 \%(9,10)$. De acuerdo a las estimaciones enunciadas en el Plan decenal para el control del cáncer en Colombia 2012-2021, se observa una disminución de la mortalidad del cáncer de cérvix en los últimos años, pero continúa el cáncer de mama en las mujeres y el de cuello uterino, siendo las dos principales causas de prevalencia y mortalidad(11).

Varios autores han reportado diversos factores de riesgo asociados al carcinoma cervical, como tener antecedentes patológicos familiares de cáncer, la menarquia precoz, la actividad sexual, en los cuales se incluye el comienzo de las relaciones sexuales en edades tempranas (antes de los 17 años)(2), la multiparidad, el cambio frecuente de compañero de actividad sexual, las infecciones de transmisión sexual, $\mathrm{PVH}(1,12)$; la literatura reporta otros factores a asociados a las dificultades para un diagnóstico oportuno como vivir en zonas alejadas o no contar con seguridad social(11,13).

La citología es el examen tamiz recomendado para la prevención del cáncer de cuello uterino $(8,10,11)$, pero en Colombia a pesar que el $99,4 \%$ de las mujeres conocen la citología, no todas se la realizan: solo el $66,5 \%$ se ha realizado la citología una vez al año, el $6,4 \%$ cada tres años y $7,7 \%$ una vez en la vida(13). Los reportes de las citologías son diversos como diversos sus factores de riesgo. Un estudio realizado en la ciudad de Cartagena reportó en sus resultados citológicos, la lesión intraepitelial de bajo grado (LIEBG) como la alteración más frecuente con el $78 \%{ }^{(14)}$. Solis y Briones reportan una prevalencia de $4,5 \%$ de lesiones intraepiteliales, de las cuales 3,2\% correspondían a LIEBG y 1,3\% a lesión intraepitelial de alto grado (LIEAG) con un 95,51\%(15).

Por otro lado, el seguimiento y tratamiento no siempre son oportunos, según el Plan Decenal de Salud Pública no existe una relación entre la cobertura en tamización y la mortalidad y se presentan dificultades en el seguimiento, diagnóstico $\mathrm{y}$ tratamiento de lesiones precancerosas y cáncer(11,16). Un estudio muestra que el 33\% de las mujeres con citología alterada no habían tenido un seguimiento apropiado, especialmente las más jóvenes(12). Otro trabajo, realizado en Medellín evidencio que las mujeres con lesión intraepitelial de alto grado no habían tenido un diagnóstico oportuno o no culminaron el tratamiento en un $10,7 \%$, encontrando una oportunidad total del $52,3 \%$ con un seguimiento menor a 3 meses. El 46,4\% manifestó haber tenido inconvenientes para acceder a las citas con especialista, las razones encontradas fueron en su mayoría de tipo administrativa como demoras en la autorización de la orden o problemas con la asignación de las citas (16,9\%-7,7\% respectivamente)(17).

El conocimiento de los múltiples factores asociados y el comportamiento de las alteraciones citológicas e histológicas en las mujeres que acceden a la tamización y su seguimiento son vitales, puesto que 
permiten orientar y reorientar acciones que posibiliten un tamizaje más efectivo, para disminuir la morbimortalidad y posibilitar las actividades tendientes a garantizar la confirmación por colposcopia y biopsia de todas aquellas mujeres que así lo requieran. Por lo expuesto el objetivo propuesto fue describir las características citológicas previas al diagnóstico de cáncer de cérvix en mujeres de una institución de la ciudad de Medellín.

\section{Materiales y métodos}

Estudio transversal descriptivo. La población estuvo conformada por 834 mujeres mayores de 14 años con diagnóstico de cáncer de cérvix atendidas en una institución que se dedica a realizar confirmación diagnóstica de mujeres con citologías alteradas. Se revisaron todos los registros correspondientes a las mujeres que asistieron para confirmación diagnóstica durante el período 2012-2017. Entre los criterios de inclusión se encontraban las mujeres que hubieran asistido por primera vez a confirmación diagnóstica por alteración citológica y fueron excluidas 120 pacientes que en su registro no tuvieron completa la información de las variables de interés. Para una muestra total de 714 mujeres con diagnostico confirmado.

La información se obtuvo de la base de datos de la institución y se filtraron los registros de las pacientes con diagnóstico de cáncer de cérvix de acuerdo a los resultados de la biopsia tomada por colposcopia o conización. Se determinaron variables como: edad, residencia, indicación y resultado de citología, fecha y resultados de colposcopia, toma y resultado de biopsia, y para calcular la oportunidad en el diagnóstico se calculó el tiempo entre la realización de la citología y la colposcopia.

\section{Análisis estadístico}

El análisis de la información se realizó en el paquete estadístico SPSS 24,0 ${ }^{\circledR}$ (Inc. Chicago, IL). Se realizó una distribución de frecuencias para las variables cualitativas (residencia, grupos de edad, resultado citología, resultado colposcopia, resultado biopsia, expresadas como frecuencias absolutas y relativas y para las variables cuantitativas (edad, tiempos), previa prueba de normalidad, se calcularon medidas de resumen y dispersión, expresadas como media y desviación estándar, o mediana y rango intercuartílico.

\section{Consideraciones éticas}

Esta investigación se consideró sin riesgo, de acuerdo a la Resolución 8430 de 1993 del Ministerio de Salud y Protección Social de Colombia, y fue aprobada por el Comité de Ética de Investigación en Salud de la Universidad Pontificia Bolivariana, y según consta en el acta $\mathrm{N}^{\circ} 5$ del 27 de abril del 2017.

\section{Resultados}

Se analizaron los registros de 714 usuarias con diagnóstico de cáncer de cérvix, entre los años 2012 y 2017. La mediana de edad fue de 51 años (rango intercuartílico 42-60), el 77,5 \% pertenecían al régimen subsidiado, $58,7 \%$ residían en el Valle de Aburrá (Tabla 1).

Tabla 1. Características demográficas de 714 mujeres con diagnóstico histológico de cáncer de cérvix atendidas en la IPS. Medellín, 2012 y 2017

\begin{tabular}{lc}
\hline \multicolumn{1}{c}{ Características } & $\begin{array}{c}\text { N=714 } \\
\mathbf{n}(\%)\end{array}$ \\
\hline Edad (P25-75) & $51(42-60)$ \\
Grupos de edad & \\
$20-39$ & $140(19,6)$ \\
$40-59$ & $384(53,8)$ \\
$>60$ & $190(26,6)$ \\
Régimen & \\
Subsidiado & $553(77,5)$ \\
Contributivo & $157(22)$ \\
Particular & $1(0,1)$ \\
Sin información & $3(0,4)$ \\
Zona de residencia & \\
Valle de aburra & $419(58,7)$ \\
Urabá & $83(11,6)$ \\
Sur oeste & $43(6)$ \\
Oriente & $38(5,3)$ \\
Norte & $35(4,9)$ \\
Bajo cauca & $32(4,5)$ \\
Occidente & $27(3,8)$ \\
Nordeste & $19(2,7)$ \\
Magdalena medio & $15(2,1)$ \\
Otro departamento & $2(0,3)$ \\
Sin información & $1(0,1)$ \\
\hline &
\end{tabular}

La principal indicación para la realización de la colposcopia fue la presencia de alguna alteración citológica $(85,2 \%)$, dentro de las cuales la más frecuente fue la lesión intraepitelial de alto grado $(33,8 \%)$ y el principal cáncer diagnosticado fue el escamocelular $(70,6 \%)$. La oportunidad entre el resultado de la citología y la realización de la colposcopia tuvo una mediana de 41 días (RIQ 22-71); observando la mayor oportunidad entre los 0 a 60 días, en 68,6 \% de las usuarias (Tabla 2). 
Tabla 2. Características citológicas y clínicas de 714 mujeres con diagnóstico histológico de cáncer de cérvix

\begin{tabular}{|c|c|}
\hline Características & $\begin{array}{c}N=714 \\
\text { n (\%) }\end{array}$ \\
\hline \multicolumn{2}{|l|}{ Indicación } \\
\hline Alteración citológica & $608(85,2)$ \\
\hline Clínica & $81(11,3)$ \\
\hline Control & $25(3,5)$ \\
\hline \multicolumn{2}{|l|}{ Reporte de citología } \\
\hline LIEAG & $241(33,8)$ \\
\hline Negativa & $184(25,8)$ \\
\hline ASC-H & $72(10,1)$ \\
\hline Mixtas & $70(9,8)$ \\
\hline LIEBG & $69(9,7)$ \\
\hline Carcinoma & $43(6)$ \\
\hline ASC-US & $35(4,9)$ \\
\hline \multicolumn{2}{|l|}{ Resultado colposcopia 1} \\
\hline Adecuado & $488(68,3)$ \\
\hline Inadecuado & $226(31,7)$ \\
\hline \multicolumn{2}{|l|}{ Resultado colposcopia 2} \\
\hline Insatisfactoria & $364(51)$ \\
\hline Satisfactorio & $350(49)$ \\
\hline \multicolumn{2}{|l|}{ Resultado colposcopia 3} \\
\hline Grado 1 & $78(10,9)$ \\
\hline Grado 2 & $519(72,7)$ \\
\hline Negativa & $117(16,4)$ \\
\hline \multicolumn{2}{|l|}{ Tipo de cáncer } \\
\hline Escamocelular & $504(70,6)$ \\
\hline Adenocarcinoma & $210(29,4)$ \\
\hline \multicolumn{2}{|l|}{ Diagnóstico } \\
\hline Biopsia & $525(73,5)$ \\
\hline Conización & $189(26,5)$ \\
\hline \multicolumn{2}{|c|}{ Tiempo hasta la realización de la colposcopia } \\
\hline 0-60 días & $444(68,6)$ \\
\hline 61-120 días & $126(19,4)$ \\
\hline >120 días & $80(12,3)$ \\
\hline \multicolumn{2}{|c|}{$\begin{array}{l}\text { LIEBG: lesión intraepitelial de bajo grado, LIEAG: lesión intraepitelial de } \\
\text { alto grado, ASC-US: atipias en células escamosas de significado incierto, } \\
\text { ASC-H: atipias en células escamosas que no descartan una lesión de alto } \\
\text { grado }\end{array}$} \\
\hline
\end{tabular}

\section{Discusión}

El propósito de este estudio fue describir las características citológicas en mujeres con diagnóstico de cáncer de cérvix. En nuestro estudio se identificó que la mayoría de las mujeres con cáncer de cérvix, tienen una edad por debajo de los 60 años; situación que es esperada con la historia natural de la enfermedad y la exposición a la infección por el Virus del Papiloma Humano (PVH), como principal agente que produce cambios histológicos en el cérvix. Otros estudios también han identificado mujeres con este cáncer por debajo de los 60 años(18-23).

Otro elemento importante a resaltar, es que la mayoría de las mujeres pertenecían al régimen subsidiado de salud, que aunque si bien, este no se considera un factor de riesgo, las condiciones sociales y económicas que subyacen a la pertenencia a este régimen de salud, como la pobreza, ciertas prácticas sexuales y la multiparidad, están relacionadas con un mayor riesgo de exposición a la infección por el PVH, debido al recambio de parejas sexuales que se pueden dar en estos contextos sociales, aunado al acceso limitado para los programas de tamización y seguimiento de la patología cervical(24).

Cabe anotar que el cáncer de cérvix, en su comportamiento histológico se considera como un cáncer progresivo, es decir, previo al establecimiento del carcinoma, se presentan estadios que empiezan desde las alteraciones citológicas y terminan en las neoplasias intraepiteliales. En este sentido, se considera para este estudio, que la mayoría de las mujeres tuvieran un resultado citológico como LIEAG y como ASC-H, considerándose estas dos alteraciones citológicas como precursoras de neoplasias intracervicales grado II o III(12,25-27).

La situación previa pone de manifiesto la importancia que tiene para los programas de tamización de cáncer de cérvix, ser oportunos en la remisión a confirmación diagnóstica de mujeres, cuya citología este reportada con alguna de las alteraciones mencionadas previamente. Aunque todas las mujeres con alteraciones citológicas de acuerdo a la ruta integral de atención en salud de mantenimiento y promoción de la salud(28) deben ser remitidas para confirmación diagnóstica dentro de los 30 días siguientes al resultado de la citología, es fundamental considerar tiempos más cortos para mujeres cuyas citologías sean reportadas como LIEAG y ASC-H.

Aunque la citología convencional es el método que más se utiliza para la tamización de cáncer de cuello uterino, y sigue siendo recomendada dentro de las atenciones del sistema de salud colombiano, se viene sugiriendo la implementación de la tamización con la prueba de ADN-PVH, para mujeres mayores de 30 años y con una frecuencia quinquenal, dado que la sensibilidad y la especificidad de esta prueba es superior a la de la citología convencional(28).

Respecto a la oportunidad entre la citología y la colposcopia/biopsia como método de confirmación diagnóstica, encontramos en nuestro estudio que la mitad de las mujeres acceden en un tiempo menor a 41 días y aproximadamente tres cuartas partes de la muestra de estudio acceden en los primeros dos meses después del resultado de la citología. Como ya 
se indicó previamente, el Ministerio de Salud considera una oportunidad adecuada los primeros treinta días, adicionalmente, el programa de tamización del Reino Unido como uno de los más importantes del mundo, recomienda una confirmación diagnóstica después de la citología alterada en un periodo no mayor a un mes(29). Otros estudios han identificado tiempos de oportunidad menores a 30 días, con ciertas variabilidades relacionadas especialmente con régimen de afiliación a salud(17,30).

Aunque el resultado de la citología es relevante, la confirmación diagnóstica es fundamental para el cáncer de cérvix. La colposcopia que es el método a través del cual se logran identificar alteraciones macroscópicas en el cuello del útero y de esta forma determinar la necesidad de tomar biopsia o no, se convierte en una consulta y paso definitivo hacia la resolución de la situación de las mujeres con alteraciones citológicas. Dentro los resultados de la colposcopia, los hallazgos colposcópicos anormales, especialmente el grado 2 , se han relacionado con la presencia de cáncer de cérvix(31), situación que queda evidenciada en nuestro estudio, donde tres cuartas partes de las mujeres presentaban este resultado en la realización de su colposcopia.

De otro lado, cabe indicar que, aunque la colposcopia es un examen fundamental en el diagnóstico del cáncer de cérvix, este procedimiento al igual que la lectura de la citología es dependiente del observador. Esto implica que los profesionales que se dedican a cualquiera de estas dos actividades, deben contar con una formación y un entrenamiento específico que los habilite para garantizar unos resultados concordantes con la situación real de la mujer. Esta situación se evidencia en este estudio al encontrar resultados de citologías negativos y hallazgos colposcópicos negativos. Aunque en Colombia no existe una certificación para la realización de la colposcopia, es importante considerarla para mejorar los indicadores de morbilidad y mortalidad relacionados con el cáncer de cérvix.

Finalmente, el cáncer más diagnosticado fue el escamocelular, proveniente de las estructuras escamosas del exocérvix, situación esperada debido a que la zona de transformación que es el lugar donde se presenta el mayor porcentaje de cáncer de cérvix, se encuentra en el exocérvix en la mayoría de los años de vida reproductiva de las mujeres(3).
Como limitaciones de este estudio, encontramos que, aunque la muestra fue grande, la base de datos no contaba con algunas variables clínicas tales como antecedentes personales relacionadas con prácticas sexuales y el estatus de infección con $\mathrm{PVH}$, que hubieran sido importantes analizar para encontrar otros factores que pudieran estar relacionados con las anomalías encontradas en los resultados de las citologías.

\section{Conclusiones}

Las características citológicas previas al diagnóstico de cáncer de cérvix tales como el LIEAG y el ASC-H, se consideran etapas previas al diagnóstico, de ahí la importancia de mejorar los programas de tamización de cáncer de cérvix y el seguimiento a las usuarias en las fases de confirmación diagnóstica y manejo.

Es necesarios realizar más estudios relacionados, teniendo en cuenta otras variables clínicas y sociodemográficas que puedan servir para realizar con mayor validez y precisión estimaciones de esta problemática, con lo cual se pudiera acercar mejor a la realidad en Colombia, aunque la incidencia ha disminuido, el cáncer de cuello uterino, sigue ocupando el segundo lugar entre las principales causas de mortalidad en las mujeres.

\section{Agradecimientos}

A la Unidad Video Diagnóstica de la Mujer, por facilitarnos el acceso a la base de datos para poder desarrollar este proyecto

Conflicto de intereses: los autores declaran no tener conflictos de interés.

\section{Referencias}

1. Montero Lora $Y$, Ramón Jimenez R, Valverde Ramón C, Escobedo Batista FE, Hodelín Pozo E. Principales factores de riesgo en la aparición del cáncer cervicouterino. MediSan. 2018;22(05):531-7. Disponible en: https://www.medigraphic.com/pdfs/medisan/mds2018/mds185j.pdf

2. Cabrera Guerra II, Ortiz Sánchez Y, Suárez Gómez YL, Socarrás Rodríguez R, Vázquez Rosales N. Factores de riesgo asociados al cáncer cérvico-uterino en el área de salud de Santa Rita. Multimed. 22 de febrero de 2016;20(5):110-28. Disponible en: https://www.medigraphic.com/pdfs/multimed/mul2016/mul165i.pdf

3. Organización Mundial de la Salud. Control integral del cáncer cervicouterino: Guía de prácticas esenciales [Internet]. WHO. 2016 [citado 21 de agosto de 2019]. Disponible en: http://www.who.int/reproductivehealth/publications/canc ers/cervical-cancer-guide/es/ 
4. Marañón Cardonne T, Mastrapa Cantillo K, Flores Barroso Y, Vaillant Lora L, Landazuri Llago S. Prevención y control del cáncer de cuello uterino. Correo Científico Méd. marzo de 2017;21(1):187-203. Disponible en: http://scielo.sld.cu/pdf/ccm/v21n1/ccm15117.pdf

5. Hernández Millan ZL, Bravo Polanco E, Águila Rodriguez N, Torres López Y, Tay Araujo JI, Martínez Santana M. Nivel de conocimientos y factores de riesgo predisponentes de cáncer cérvico-uterino en mujeres de Cumanayagua. Rev Cuba Enferm. junio de 2016;32(2):141-50. Disponible en: http://scielo.sld.cu/scielo.php?script=sci_arttext\&pid=S086 4-03192016000200001

6. Bray F, Ferlay J, Soerjomataram I, Siegel RL, Torre LA, Jemal A. Global cancer statistics 2018: GLOBOCAN estimates of incidence and mortality worldwide for 36 cancers in 185 countries. CA Cancer J Clin. 2018;68(6):394-424. https://doi.org/10.3322/caac.21492

7. Global Cancer Observatory [Internet]. Lyon: IARC; 2020 [citado 2019 oct 7]. Disponible en: http://gco.iarc.fr/

8. Organización Mundial de la Salud. Cáncer Cervicouterino [Internet]. Pan American Health Organization / World Health Organization. 2018 [citado 24 de septiembre de 2019]. Disponible

en: https://www.paho.org/hq/index.php?option=com_content \&view=article \&id=5420:2018-cervical-

cancer\&Itemid $=3637$ \&lang=es

9. World Health Organization. Global Cancer Observatory: Colombia. [Internet]. 2018 [citado 24 de septiembre de 2019]. Disponible

en: https://gco.iarc.fr/today/data/factsheets/populations/170 -colombia-fact-sheets.pdf

10. Cáncer de cuello uterino [Internet]. Bogotá: Minsalud; 2020 [citado 24 de septiembre de 2019]. Disponible en: https://www.minsalud.gov.co/salud/publica/ssr/Paginas/ Cancer-de-cuello-uterino.aspx

11. Ministerio de salud y Protección Social, Instituto Nacional de Cancerología. Plan decenal para el control del cáncer en Colombia, 2012-2021. 2 Ed. [Internet]. 2012 [citado 12 de septiembre de 2018]. Bogotá D.C. Disponible en: https://www.minsalud.gov.co/sites/rid/Lists/BibliotecaDi gital/RIDE/IA/INCA/plan-nacional-control-cancer.pdf

12. Doris B, Boyer L, Lavoué V, Riou F, Henno S, Tas P, et al. Cervical Papsmear in an epidemiologically exposed population: ideas, facts and arguments. J Gynecol Obstet Biol Reprod (Paris). 2014;43(1):26-34. https://doi.org/10.1016/j.jgyn.2013.11.012

13. Ministerio de Salud, Profamilia. Encuesta Nacional de Demografía y Salud, Tomo II: Componente de Salud Sexual y Salud Reproductiva [Internet]. 2015 [citado 23 de mayo de 2018]. Disponible

https://profamilia.org.co/investigaciones/ends/

14. Barrios García L, Lecompte Osorio PA, Leones Castillo RA, López Custode FR. Factores de riesgo presentes en pacientes con lesiones intraepiteliales escamosas del cérvix en la Clínica Maternidad Rafael Calvo en la ciudad de Cartagena (Colombia): estudio descriptivo. Arch Med Col. 2016;16(1):109-17. Disponible en: https://www.redalyc.org/pdf/2738/273846452011.pdf

15. Solis JG, Briones-Torres TI. Prevalencia de lesión intraepitelial en citología cervical de tamizaje en una unidad de primer nivel de atención. Rev Médica Inst Mex Seguro Soc. 21 de agosto de 2018;56(2):167-72. Disponible en: https://www.medigraphic.com/pdfs/imss/im2018/im182j.pdf
16. Garcés Palacio I, Rocha Buelvas A. Barreras para la detección oportuna del cáncer cervicouterino en Colombia: una revisión narrativa. Rev Investigaciones Andina En Línea. 13 de octubre de 2017;18(33):1647-64. Disponible en: https://revia.areandina.edu.co/index.php/IA/article/view/ $648 / 584$

17. Valencia-Arredondo $M$, Herrera-Posada D, MontoyaRodríguez D, Navales-Rojas J. Seguimiento a mujeres con resultado citológico positivo en una Empresa Social del Estado. Medellín, Colombia. 2011- 2012. Rev Fac Nac Salud Pública. enero de 2017;35(1):27-38. http://dx.doi.org/10.17533/udea.rfnsp.v35n1a04

18. Fernández-Mercado R, Amaya-Guío J, González-Rubio Á, Pineda-Vega R, Riveros-Torrado E, Álvarez-González A, Corro-Melgarejo JM. Características clínicas y pronóstico de las pacientes con cáncer de cérvix tratadas en tres instituciones de salud en Barranquilla, Colombia, de 2005 a 2011. Rev Colomb Obstet Ginecol. junio de 2014;65 (2):1208. https://doi.org/10.18597/rcog.60

19. Reyes Zuñiga YI, Sanabria Negrín JG, Marrero Fernández R. Incidencia y mortalidad por cáncer cervicouterino. Rev Ciencias Médicas. octubre de 2014;18 (5):753-66. Disponible en: http://scielo.sld.cu/pdf/rpr/v18n5/rpr05514.pdf

20. Dávila Gómez HL, Matos Rodríguez Z, Esquivel Díaz O, López Ruiz M. Cervical cancer in patients without abnormal cytology. Youth island, study by four years. J Pregnancy Reprod. http://dx.doi.org/10.15761/JPR.1000133

21. Gyenwali D, Pariyar J, Raj Onta S. Factors Associated with Late Diagnosis of Cervical Cancer in Nepal. Asian Pacific Journal of Cancer Prevention. 2013; 14(7):4373-7. http://dx.doi.org/10.7314/apjcp.2013.14.7.4373

22. Fondo Colombiano de Enfermedades de Alto Costo-Cuenta de Alto Costo [CAC]. Situación del cáncer en la población adulta atendida en el SGSSS de Colombia 2018 [Internet]. Bogotá, D.C.; 2019 [citado 25 de agosto de 2020]. Disponible en: https://cuentadealtocosto.org/site/wpcontent/uploads/2019/10/Libro_Situacion_Cancer_2018_c ompressed-1.pdf

23. González TM. Abordaje, diagnóstico y tratamiento de las lesiones premalignas de cérvix en la consulta externa del Hospital Alemán Nicaragüense en el periodo comprendido de enero 2014 a diciembre 2015. [Managua]: Universidad Nacional Autónoma de Nicaragua; 2016. Disponible en: https://repositorio.unan.edu.ni/1550/1/58908.pdf

24. Oliveira Nicolau AI, de Souza Aquino P, Barbosa Ximenes L, Bezerra Pinheiro K. Proximal social determinants related to cervical cancer in imprisioned women. Rev Min Enferm. 2015;19 3):733-40. http://dx.doi.org/10.5935/14152762.20150055

25. Ministerio de Salud, Dirección General de Promoción y Prevención. Norma técnica para la detección temprana del cáncer de cuello uterino y guía de atención de lesiones preneoplásicas de cuello uterino [Internet]. 2000 [citado 20 de abril de 2018]. Disponible en: http://odm.colnodo.apc.org/apc-aafiles/5774d034c9be96efe0bff91311203bc4/Norma_tecnica _para_la_deteccion_temprana_del_cancer_de_cuello.pdf

26. Pereira ER, Speck NM de G, Rodrigues DA, De Freitas VG, Ribalta JCL. Prevention, diagnosis and treatment of cervical cancer precursor lesions at the Xingu Indigenous Park, Brazil. Eur J Gynaecol Oncol. 2015; 36 (4):376-82. Disponible en: https://pubmed.ncbi.nlm.nih.gov/26390686/ 
27. Torres Lobatón A, Bustamante Iglesias JI, Torres Rojo A, Oliva Posada JC, Morales Palomares MÁ, Román Bassaure E. Cáncer cervicouterino. Perfil epidemiológico en 1,217 pacientes. Seguro popular. Ginecol Obstet México. 2013;81 (2):71-6. Disponible en: https://www.medigraphic.com/pdfs/ginobsmex/gom2013/gom132b.pdf

28. Ministerio de Salud y Protección Social. Resolución 3280 de 2018 [Internet]. Bogotá, D.C. 2018 [citado 9 de octubre de 2019]. Disponible en: https://www.minsalud.gov.co/sites/rid/Lists/BibliotecaDi gital/RIDE/DE/DIJ/resolucion-3280-de-2018.pdf

29. Public Health England. Cervical Screening Programme Colposcopy and Programme Management [Internet]. London: Cambrian Printers; 2010 [citado 9 de octubre de 2019]. Disponible en: https://www.gov.uk/government/publications/cervicalscreening-programme-and-colposcopymanagement\#history

30. Agudelo Hincapie KJ, García Carvajal DL, Cardona Arias JA, Valencia Arredondo M. Seguimiento de pacientes con atipia de células escamosas de significado indeterminado (ASCUS) en un servicio citológico de Medellín (Colombia), 2011-2015. Medicina \& Laboratorio. 2016;22 (9-10):447-58. https://doi.org/10.36384/01232576.93

31. Nghiem VT, Davies KR, Beck JR, Follen M, Cantor SB. Overtreatment and cost-effectiveness of the see-and-treat strategy for managing cervical precancer. Cancer Epidemiol Biomark Prev Publ Am Assoc Cancer Res Cosponsored Am Soc Prev Oncol. mayo de 2016;25(5):807-14. https://doi.org/10.1158/1055-9965.EPI-15-1044 